\title{
Blocking Borrelia burgdorferi transmission from infected ticks to nonhuman primates with a human monoclonal antibody
}

\author{
Zachary A. Schiller, ${ }^{1}$ Michael J. Rudolph, ${ }^{2}$ Jacqueline R. Toomey, ${ }^{1}$ Monir Ejemel, ${ }^{1}$ Alan LaRochelle, ${ }^{3}$ Simon A. Davis, ${ }^{2}$ \\ Havard S. Lambert, ${ }^{4}$ Aurélie Kern, ${ }^{5}$ Amanda C. Tardo, ${ }^{4}$ Colby A. Souders, ${ }^{1}$ Eric Peterson, ${ }^{1}$ Rebecca D. Cannon, \\ Chandrashekar Ganesa, ${ }^{1}$ Frank Fazio, ${ }^{1}$ Nicholas J. Mantis, ${ }^{6}$ Lisa A. Cavacini, ${ }^{1}$ John Sullivan-Bolyai, ${ }^{1}$ Linden T. Hu, ${ }^{5}$ \\ Monica E. Embers, ${ }^{4}$ Mark S. Klempner, ${ }^{1}$ and Yang Wang ${ }^{1}$ \\ 'MassBiologics of the University of Massachusetts Medical School, Boston, Massachusetts, USA. ${ }^{2}$ New York Structural Biology Center, New York, New York, USA. ${ }^{3}$ Biomere, Worcester, Massachusetts, USA. \\ ${ }^{4}$ Division of Bacteriology and Parasitology, Tulane National Primate Research Center, Tulane University Health Sciences Center, Covington, Louisiana, USA. ${ }^{5}$ Sackler School of Graduate Biomedical Sciences, \\ Tufts University School of Medicine, Boston, Massachusetts, USA. Division of Infectious Disease, Wadsworth Center, New York State Department of Health, Albany, New York, USA
}

Disrupting transmission of Borrelia burgdorferi sensu lato complex (B. burgdorferi) from infected ticks to humans is one strategy to prevent the significant morbidity from Lyme disease. We have previously shown that an anti-OspA human mAb, 2217, prevents transmission of $B$. burgdorferi from infected ticks in animal models. Maintenance of a protective plasma concentration of a human mAb for tick season presents a significant challenge for a preexposure prophylaxis strategy. Here, we describe the optimization of mAb 2217 by amino acid substitutions (2217LS: M428L and N434S) in the Fc domain. The LS mutation led to a 2-fold increase in half-life in cynomolgus monkeys. In a rhesus macaque model, 2217LS protected animals from tick transmission of spirochetes at a dose of $3 \mathrm{mg} / \mathrm{kg}$. Crystallographic analysis of Fab in complex with OspA revealed that 2217 bound an epitope that was highly conserved among the B. burgdorferi, B. garinii, and B. afzelii species. Unlike most vaccines that may require boosters to achieve protection, our work supports the development of $2217 \mathrm{LS}$ as an effective preexposure prophylaxis in Lyme-endemic regions, with a single dose at the beginning of tick season offering immediate protection that remains for the duration of exposure risk.

\section{Introduction}

Lyme disease is a bacterial infection that is transmitted through the bite of infected Ixodes ticks. The vast majority of cases of Lyme disease occur during the spring, summer, or fall and are caused by questing ticks carrying Borrelia burgdorferi sensu lato complex (B. burgdorferi). Lyme disease in humans is characterized by erythema migrans (EM); fever; fatigue; musculoskeletal pain; and the potential for neurological, cardiac, or joint manifestations. The CDC has estimated that over 476,000 Americans are diagnosed with and treated for Lyme disease each year $(1,2)$. In Europe, approximately 85,000 Lyme disease cases occur annually (3). Worldwide, 3 main species of B. burgdorferi sensu lato complex are associated with Lyme disease in humans. B. burgdorferi is the main cause of Lyme disease in North America; B. garinii and B. afzelii are the prevalent strains that cause the disease in European and Asian countries (4). Humans can become infected by nymphs or less commonly adult ticks that are infected with B. burgdorferi (5).

Authorship note: MSK and TW are co-corresponding authors.

Conflict of interest: MSK and YW are named as coinventors on a relevant patent: Anti-OspA Antibody and Methods of Use, US patent 10,457,721.

Copyright: @ 2021, American Society for Clinical Investigation.

Submitted: October 5, 2020; Accepted: April 22, 2021; Published: June 1, 2021

Reference information: J Clin Invest. 2021;131(11):e144843.

https://doi.org/10.1172/JCl144843.
Transmission of B. burgdorferi from ticks to vertebrate hosts can be blocked in animal models by polyclonal antibodies raised against the bacterial outer surface protein $\mathrm{A}(\mathrm{OspA})$ as well as by a mAb (LA-2) directed against a specific OspA epitope (6). Based on the effectiveness of OspA-specific humoral immunity in animal models, human vaccines containing recombinant OspA of B. burgdorferi were developed for the prevention of Lyme disease. Large-scale clinical trials demonstrated the effectiveness of a triple-dose OspA vaccine that protected up to $92 \%$ of human volunteers (3). However, the vaccine was voluntarily removed from the market for multiple reasons, including the low risk of Lyme disease in many parts of the country, the need for repeated injections, the relatively high cost compared with antibiotic treatment during early infection, a middling endorsement by the public health community, and potential safety concerns over reactivity with a hypothetical arthritogenic portion of the OspA protein. No vaccine is currently available to prevent human Lyme disease, although there is a vaccine candidate in phase II clinical trials (7-9). Furthermore, public acceptance of a Lyme vaccine is uncertain, given the failure of prior efforts and prevailing skepticism regarding vaccines. Thus, there is an urgent need to develop a novel approach for prevention of Lyme disease.

We developed a panel of anti-OspA human mAbs (HuMAbs) as potential prophylaxis for Lyme disease (10). In particular, HuMAb 2217 was shown to be borreliacidal $\left(\mathrm{EC}_{50}<1 \mathrm{nM}\right)$ against $B$. burg- 
A

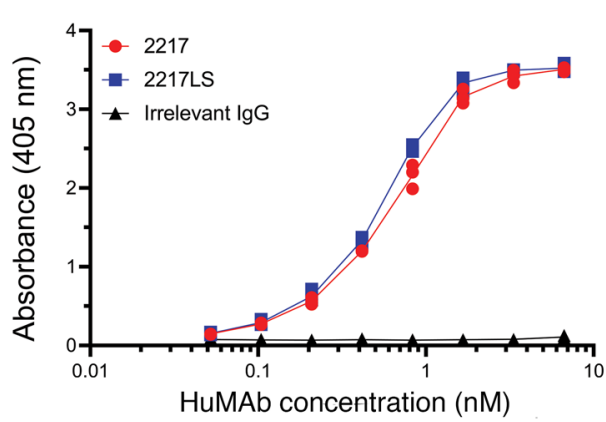

C

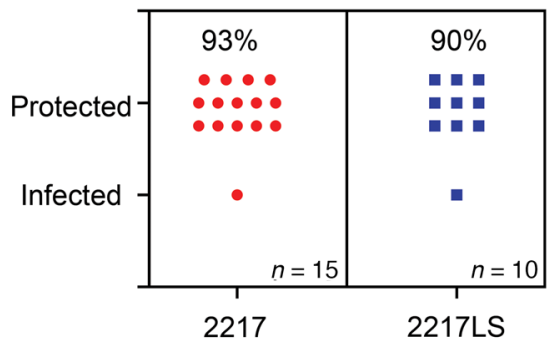

B

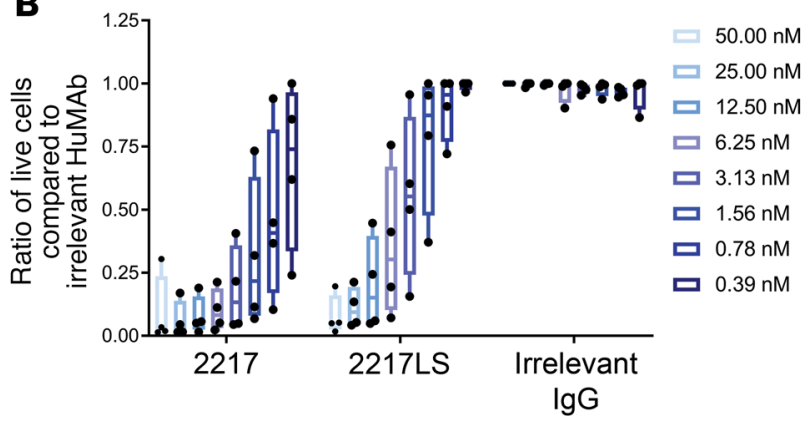

Figure 1. Efficacy characterization of 2217LS. (A) The binding activity of 2217 (red circles) and 2217LS (blue squares) was determined by ELISA against $B$. burgdorferi outer surface protein $\mathrm{A}(\mathrm{OspA})$ using ELISA. The $\mathrm{EC}_{50}$ values were calculated and represent the concentration of $\mathrm{mAb}$ required for a $50 \%$ reduction in absorbance measured at $405 \mathrm{~nm}$. Binding data are plotted as $n=3$ independent studies. (B) The borreliacidal activity of 2217 and $2217 \mathrm{LS}$ was determined by incubating 2217 and 2217LS ( $0.4 \mathrm{nM}$ to $50 \mathrm{nM}$ ) with $B$. burgdorferi spirochetes. The viability of spirochetes was quantified by luciferase detection and normalized against an irrelevant IgC control. The percentages of live spirochetes were plotted as $n=4$ independent studies. The $\mathrm{EC}_{50}$ for $B$. burgdorferi increased from $0.48 \pm 0.32 \mathrm{nM}$ for 2217 to $3.71 \pm 2.81 \mathrm{nM}$ for $2217 \mathrm{LS}$. Whiskers represent minimum and maximum. Boxes represent IQR. Line represents the median, and dots represent data points ( $n=4$ for each group). (C) The in vivo efficacy was determined by challenging mice that were administered $5 \mathrm{mg} /$ $\mathrm{kg}$ of either 2217 or $2217 \mathrm{LS}$. The results indicated that $90 \%$ of mice $(n=10)$ treated with $2217 \mathrm{LS}$ were protected, which is comparable to the $93 \%$ of mice $(n=15)$ that were protected by 2217. Protection comparisons were conducted using 2-sided Fisher's exact test.

dorferi, B. afzelii, and B. garinii, the 3 main genospecies endemic in the United States, Europe, and Asia. When passively administered to mice prior to challenge with B. burgdorferi-infected Ixodes ticks, HuMAb 2217 completely prevented transmission after a single dose of $10 \mathrm{mg} / \mathrm{kg}$.

One challenge to developing 2217 for Lyme disease prevention is to ensure that the antibody sustains a protective plasma concentration for the entire tick season. Although typical human IgG1 antibodies have an average half-life of 21 days, advances in antibody modification over the last decade have dramatically extended antibody half-life (11). Antibody IgG half-life is determined by its interaction with the neonatal $\mathrm{Fc}$ receptor $(\mathrm{FcRn})$, which binds IgG in a $\mathrm{pH}$-dependent manner and protects it from degradation. Mutations that prolong antibody half-life were identified by modulating antibody Fc domain binding to FcRn. Specifically, the YTE (M252Y, S254T, and T256E) and LS (M428L and N434S) mutations demonstrated significant extension of antibody half-life in animal and human studies (12-16). It was recently reported that an LS-mutated antibody against HIV (VRC01LS) resulted in sustained serum concentration of 10 $\mu \mathrm{g} / \mathrm{mL}$ over 5 months after a single $5 \mathrm{mg} / \mathrm{kg}$ dose $(15,16)$. Thus, we reason that, unlike the previously approved Lyme vaccine (LYMERix), which required repeat boosters to achieve immunity, administration of a single dose of a half-life-extended antiOspA human $\mathrm{mAb}$ at the beginning of the tick season could be sufficient for protection during the period of risk in Lymeendemic regions of North America.
In this study, we engineered half-life extension mutations M428L and N434S in antibody 2217 (2217LS) and characterized antibody pharmacokinetics and efficacy in mice and nonhuman primate models of Lyme disease. Our study demonstrated that 2217LS had an extended half-life and was able to prevent the transmission of B. burgdorferi from ticks to mice and nonhuman primates.

\section{Results}

Engineering and efficacy characterization of 2217LS. We previously developed a panel of anti-OspA IgG HuMAbs that demonstrated strong efficacy against tick transmission of B. burgdorferi in a murine challenge model. The lead candidate, 2217, protected mice against spirochete transmission during an infected tick blood meal at a dose of $5 \mathrm{mg} / \mathrm{kg}$ (10). To extend the antibody half-life of 2217, LS (M428L and N434S) mutations were engineered into the Fc domain by site-directed mutagenesis. ELISA and a borreliacidal assay were conducted to evaluate in vitro functional activities of 2217LS. The $\mathrm{EC}_{50}$ for B. burgdorferi OspA in ELISA was comparable between $2217(0.47 \pm 0.01 \mathrm{nM})$ and $2217 \mathrm{LS}\left(0.56 \pm 0.03 \mathrm{nM}\right.$ ) (Figure 1A), and the $\mathrm{EC}_{50}$ for B. burgdorferi in the borreliacidal assay increased from $0.48 \pm 0.32 \mathrm{nM}$ for 2217 to $3.71 \pm 2.81 \mathrm{nM}$ for 2217LS (Figure 1B). To determine whether 2217LS retained in vivo efficacy despite the slightly reduced in vitro potency, 2217 and 2217LS were compared in a murine challenge model. HuMAb 2217 or 2217LS was administered to $\mathrm{C} 3 \mathrm{H} / \mathrm{HeJ}$ mice challenged with $B$. burgdorferi-infected 
A

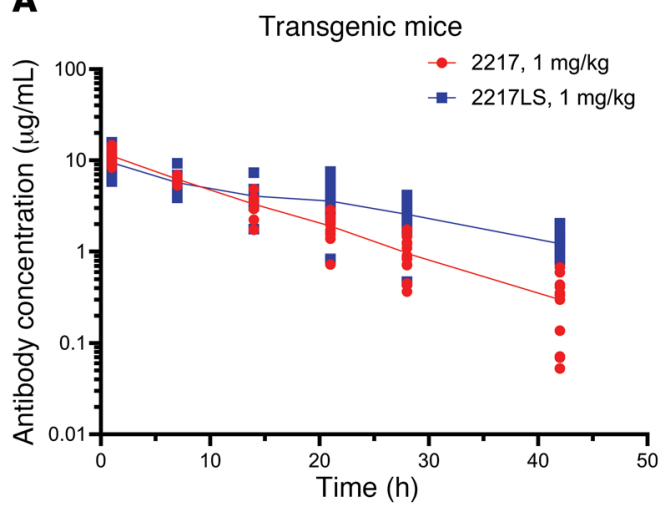

B

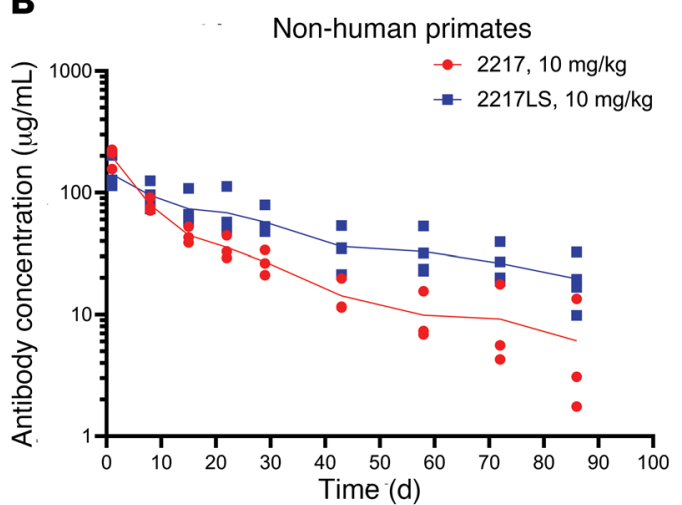

Figure 2. LS mutation extends half-life of 2217 in FcRn mice and nonhuman primates. Observed 2217 (red circles) and 2217LS (blue squares) serum pharmacokinetic profile after a single dose of (A) $1 \mathrm{mg} / \mathrm{kg}$ in transgenic FcRn mice ( $n=16$ per group) and (B) $10 \mathrm{mg} / \mathrm{kg}$ in cynomolgus monkeys ( $n=$ 4 per group). The LS mutation reduced 2217 clearance in both species, leading to an observed 2 -fold increase in half-life in both species (mouse $P=$ 0.0105 ; nonhuman primate $P=0.006$ ). Half-life comparisons were conducted using 2 -sided Student's $t$ test. Symbols represent the means for each animal tested in $n=3$ independent assays.

nymphs. Efficacy was determined by preventing spirochete transmission between nymphs and the mouse (Supplemental Table 1; supplemental material available online with this article; https:// doi.org/10.1172/JCI144843DS1). The results indicated that 9 of 10 mice (90\%) treated with $5 \mathrm{mg} / \mathrm{kg} 2217 \mathrm{LS}$ were protected, which is comparable to 14 of 15 mice (93\%) that were protected by 2217 ( $P>0.99$; Figure 1C). Overall, these results indicated that the slight reduction of in vitro bactericidal activity observed with the LS mutation did not affect the in vivo protective efficacy of 2217.

LS mutation extends half-life in FcRn-transgenic mice. To assess the change in pharmacokinetics conferred by the LS mutations, 2217LS was compared with 2217 in a human FcRn-transgenic mouse model that provides a relative comparison for half-life differences in humans. These animals lack the endogenous mouse FcRn $\alpha$ gene and express the human FcRn $\alpha$ gene on 1 allele under control of the native FcRn promoter (17). Human FcRn-transgenic mice $(n=10)$ received a single i.p. injection containing a collection of mAbs with known human half-lives as well as 2217 or 2217LS administered at $1 \mathrm{mg} / \mathrm{kg}$. Serum was collected over several days and used to determine antibody concentration (Figure 2A). Half-life was then calculated by noncompartmental analysis (NCA) using Phoenix WinNonlin software. Relative to 2217, the LS modification extended the half-life of 2217 by 2.0 times, from $8.0 \pm 0.4$ days to $16.0 \pm 1.1$ days $(P=0.0105)$.

LS mutation extends half-life in nonhuman primates. Nonhuman primates represent a closer model to humans than FcRn-transgenic mice, allowing for more predictive half-life modeling. Cynomolgos monkeys were administered $2217(n=4)$ or 2217LS ( $n=4)$ by i.v. injection at $10 \mathrm{mg} / \mathrm{kg}$ (Figure 2B). Serum was collected over the course of 86 days and assayed by ELISA to determinate the concentration of the antibodies in the serum. These concentrations were used to calculate half-lives by NCA using Phoenix WinNonlin software. Relative to 2217, the LS modification extended the half-life of 2217 by 2.1 times, from $15.41 \pm$ 7.5 days to $31.8 \pm 2.5$ days $(P=0.006)$. The 2 -fold increase aligns with our data in FcRn mice and other nonhuman primate studies involving half-life extension with the LS modification (16).
2217LS blocks transmission of B. burgdorferi in a nonhuman primate model. To determine the dose required for protection, 2217LS was tested in a recently developed nonhuman primate tick transmission model. This nonhuman primate model closely mimics the transmission, pathology, and progression of Lyme disease in humans $(18,19)$. To ensure tick containment and feeding, we utilized a modified LeFlap tick-containment device that was attached to the primates after they were injected with anti-OspA HuMAbs. Previous nonhuman primate studies utilizing this containment method have had $90 \%$ success rates of tick feeding and B. burgdorferi transmission (19). Twenty-two rhesus macaques (Macaca mulatta) were divided into 5 treatment groups and administered either 2217LS or an irrelevant HuMAb by i.v. injection (Figure 3). 2217LS was administered at $90 \mathrm{mg} /$ $\mathrm{kg}$ (group $1 ; n=4$ ), $30 \mathrm{mg} / \mathrm{kg}$ (group $2 ; n=5$ ), $10 \mathrm{mg} / \mathrm{kg}$ (group $3 ; n=6$ ), or $3 \mathrm{mg} / \mathrm{kg}$ (group $4 ; n=4$ ). The irrelevant HuMAb was administered at $10 \mathrm{mg} / \mathrm{kg}$ (group $5 ; n=3$ ). In order to determine the level of antibody needed for protection, serum samples were collected just prior to challenge ( 24 hours after administration). The antibody concentrations in the serum for each group were proportional to the difference in dose levels (Supplemental Figure $1, A$ and B). Group 1 had a $C_{\text {max }}$ of $1122 \pm 209 \mu \mathrm{g} / \mathrm{mL}$, group 2 had a $C_{\max }$ of $390 \pm 58 \mu \mathrm{g} / \mathrm{mL}$, group 3 had a $C_{\max }$ of $147 \pm 84 \mu \mathrm{g} /$ $\mathrm{mL}$, and group 4 had a $\mathrm{C}_{\max }$ of $41 \pm 5 \mu \mathrm{g} / \mathrm{mL}$. Additional serum samples were collected after the 24-hour time point through 63 days after administration, and tissue samples were collected weekly after the ticks were removed for 4 weeks.

All animals were screened for anti-drug antibody (ADA) responses by detection of nonhuman primate IgGs against 2217LS or the irrelevant IgG. Four out of 18 of the animals had ADA responses. These animals were distributed among the 10, 30 , and $90 \mathrm{mg} / \mathrm{kg}$ groups, suggesting that the responses were not concentration dependent. Two of the animals in the irrelevant IgG group had similar ADA responses. Importantly, these responses appeared after day 7 and did not significantly affect 2217LS serum concentration during the acute challenge period (Supplemental Figure 1C). 


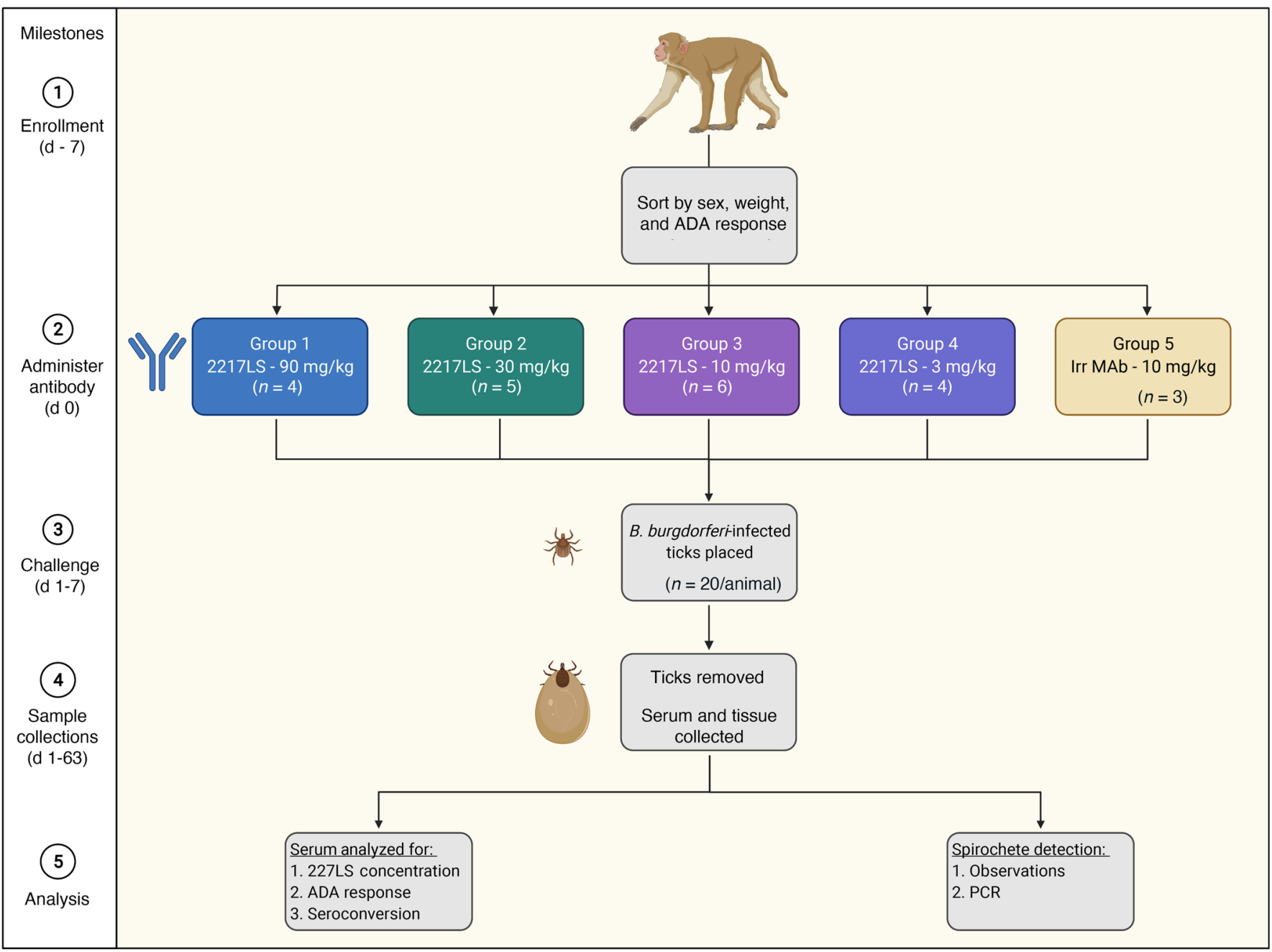

Figure 3. 2217LS blocks transmission of Borrelia burgdorferi in a nonhuman primate model. (A) Schematic of nonhuman primate tick challenge model showing time points for group assignment, antibody administration, challenge, sample collections, and analysis biopsy. Animals were assigned to 1 of 5 treatment groups based on sex, weight, and ADA response. Animals were i.v. administered 90, 30, 10, or $3 \mathrm{mg} / \mathrm{kg}$ of $2217 \mathrm{LS}$ or $10 \mathrm{mg} / \mathrm{kg}$ of an irrelevant HuMAb. Animals were challenged with $B$. burgdorferi-infected ticks 24 hours later. Ticks were given 6 days to take a blood meal. On day 7 , the ticks were collected. Tissue (days 7, 14, 21, and 28) and serum (days -7, 0, 1, 7, 14, 21, 28, 45, and 63) samples were collected and analyzed for pharmacokinetics and efficacy. Figure was created with BioRender.com.

All animals were challenged with 20 B. burgdorferi-infected nymphs. This represents a significantly greater challenge than a single tick, which would be more typical of a natural encounter in human infection. Skin biopsies were taken weekly during the first 4 weeks after infection. Biopsies were cultured in BSK-H medium for 6 weeks and observed under dark-field microscopy for the presence $(+)$ or absence $(-)$ of spirochetes (Table 1$)$. A positive culture $(+)$ was defined as the observation of motile spirochetes in consecutive weeks throughout the 6-week culture observation period. One animal in the $2217 \mathrm{LS} 10 \mathrm{mg} / \mathrm{kg}$ group and one in the irrelevant IgG group yielded culture-positive skin biopsies. In each of these animals, live spirochetes were observed in 1 biopsy among the 4 biopsies taken from animals. Further, dead or nonmotile spirochetes were observed in the skin biopsy cultures from some animals, including animals G67H and $\mathrm{J797}$ (10 mg/kg); RA3836, RA3869, and RA3875 (3 mg/kg); and G72G and J393 (control IgG) groups, but just within the first 2 weeks of the 6-week-long culture and observation period. No live spirochetes were ever observed in any cultures of these animals. Therefore, we denoted these animals as negative in Table 1 .

Skin biopsies were also assessed for spirochetal DNA. Aliquots were taken weekly from the skin biopsy cultures and analyzed by PCR using primers designed against the OspA gene of B. burgdorferi. An aliquot from an in vitro B. burgdorferi culture $\left(1 \times 10^{7}\right.$ spirochetes $/ \mathrm{mL}$ ) in BSK-H medium was used as a positive control. All weekly aliquots of the cultures with a few exceptions came back as PCR negative for the OspA gene in consecutive weeks throughout the 6-week culture observation period. The aforementioned exceptions were 3 animals in the $90 \mathrm{mg} / \mathrm{kg}$ group (RA3835, RA3896, and RA3901). Faint positive bands were found during PCR analysis of 1 of the 4 tissue biopsy cultures taken from each animal. However, these faint bands were only observed during the second week of cultures and were not found in any subsequent weekly analysis. Therefore, we denoted these animals as negative in Table 1.

Culturing spirochetes from skin biopsies has been shown to be highly variable, especially from primates (20). In the absence of 
Table 1. Detection of $B$. burgdorferi transmission in nonhuman primates

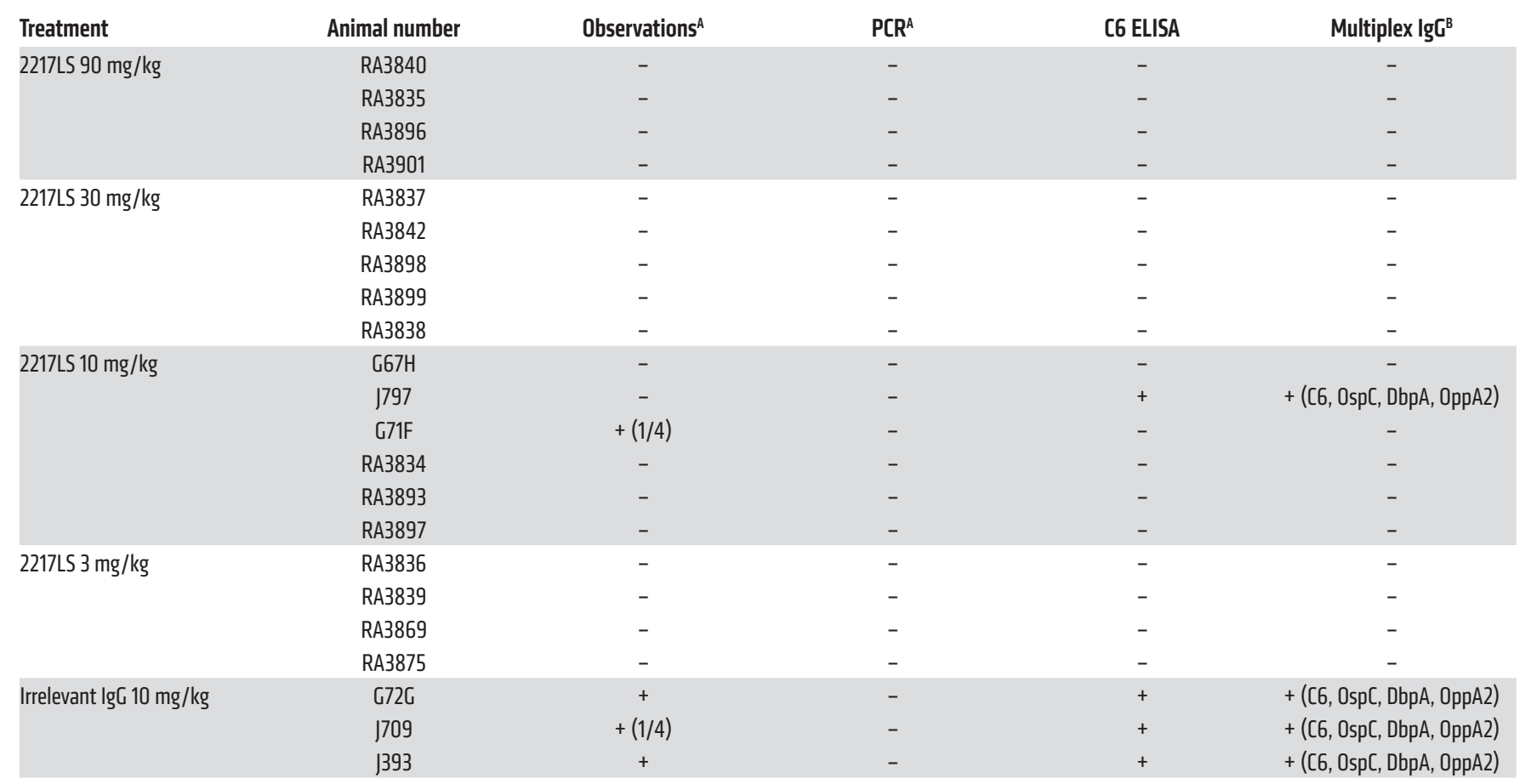

${ }^{A}$ Four tissue samples were collected over the course of 4 weeks from the tick feeding site. ${ }^{B}$ Multiplex assay was conducted against C6, OspA, OspC, DbpA, and OppA2 antigens. A "-" denotes negative and "+" denotes positive.

an EM rash, the biopsies were taken near the papules that serve as evidence of the tick bite site. In a human with EM, the biopsy would be taken from the leading edge. The skin sample collection from primates might not reflect the dispersion of spirochetes, leading to negative culture results.

Seroconversion for IgG antibodies against the B. burgdorferi antigen C6, a synthetic peptide of the invariable region 6 of VlsE (variable major protein-like sequence, expressed), was measured by ELISA to analyze transmission as an indicator of efficacy. Only one animal, in the $2217 \mathrm{LS} 10 \mathrm{mg} / \mathrm{kg}$ group, showed evidence of transmission as measured by seroconversion to $\mathrm{C} 6$ antigen. Anti-C6 IgGs increased by 21 days after tick exposure, which mirrored the seroconversion of irrelevant IgG-treated animals (Figure 4A). All remaining animals in the 3,10, 30, and $90 \mathrm{mg} /$ $\mathrm{kg}$ groups were protected, with no detectable IgG response against the C6 peptide (Figure 4A).

To confirm the C6 peptide ELISA result, a multiplex bead array assay was conducted to provide quantitative measurement of seroconversion to a series of antigens, including B. burgdorferi antigens C6 (Figure 4B), OspA (Figure 4C), outer surface protein C (OspC) (Figure 4D), decorin-binding protein A (DpbA) (Figure 4E), and oligopeptide permease A2 (OppA2) (Figure 4F). This assay has higher sensitivity and specificity than the enzyme immunoassay and the 2-tier test, which are typically used for Lyme diagnosis (21). The multianalyte analysis showed increases in IgG against OspA, DpbA, C6, OspC, and OppA2 over time (day 21 and day 63) in the control group animals as well as 1 animal in the $10 \mathrm{mg} / \mathrm{kg}$ group that showed C6 seroconversion. In contrast, all other ani- mals in the $3,10,30$, and $90 \mathrm{mg} / \mathrm{kg}$ groups were protected, with no detectable IgG response against all tested antigens. This observation is consistent with the anti-C6 ELISA data, suggesting that all 2217LS-treated groups resulted in $83 \%-100 \%$ protection.

Structure of Fab 2217 in complex with OspA. To obtain a detailed molecular understanding of the interaction between 2217 and OspA, we examined the x-ray crystal structure of Fab 2217 in complex with OspA. The Fab-OspA structure was solved at $3.0 \AA$ resolution in the C2 space group and formed a 1:1 stoichiometric complex (Figure 5A) with 2 Fab-OspA complexes in the crystallographic asymmetric unit. The 2 Fab-OspA structures within the crystallographic asymmetric unit were very similar to each other, with a root-mean-square deviation (RMSD) of $0.8 \AA$ between 667 residues from each molecule upon superposition, indicating no major structural differences between each FabOspA complex. After molecular replacement calculations were performed, the resulting phase information was used to calculate electron density maps utilized to manually insert 2217 residues into the model and manually build other regions of 2217 and OspA within the Fab 2217-OspA model. Crystallographic and refinement data for the Fab 2217-OspA structure demonstrated a refined molecular model that is consistent with the crystallographic data as well as excellent geometry (Supplemental Table 2). Fab 2217 assumed the canonical Fab structure with 2 heavy chain Ig domains $\left(\mathrm{V}_{\mathrm{H}}\right.$ and $\left.\mathrm{C}_{\mathrm{H}} 1\right)$ and 2 light Ig domains $\left(\mathrm{V}_{\mathrm{L}}\right.$ and $\left.\mathrm{C}_{\mathrm{L}}\right)$, each containing 7-9 $\beta$-strands arranged in $2 \beta$-sheets that folded into a 2-layer sandwich with all 6 complementarity-determining regions (CDRs) (L1, L2, L3, H1, H2, and H3) on 1 face of the Fab 
A

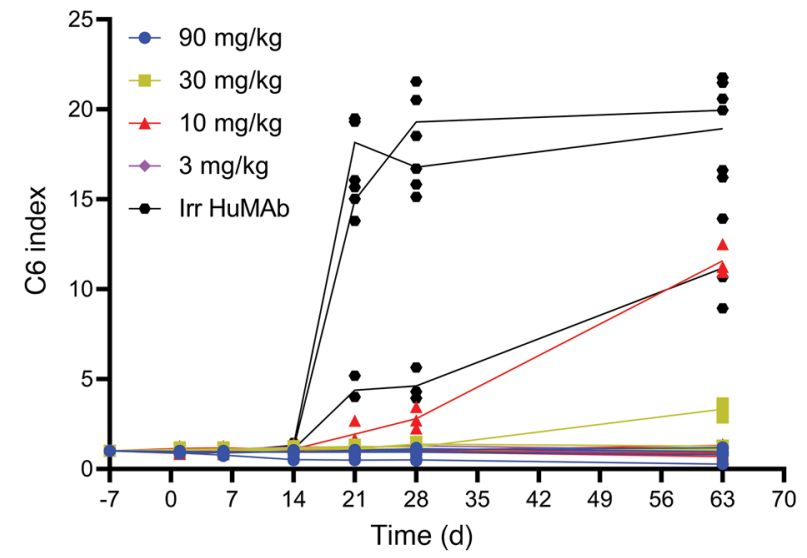

C

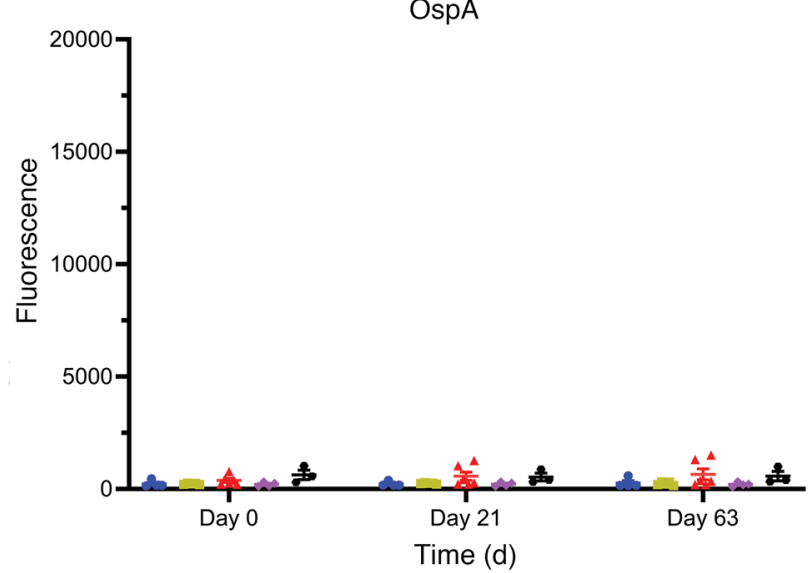

E

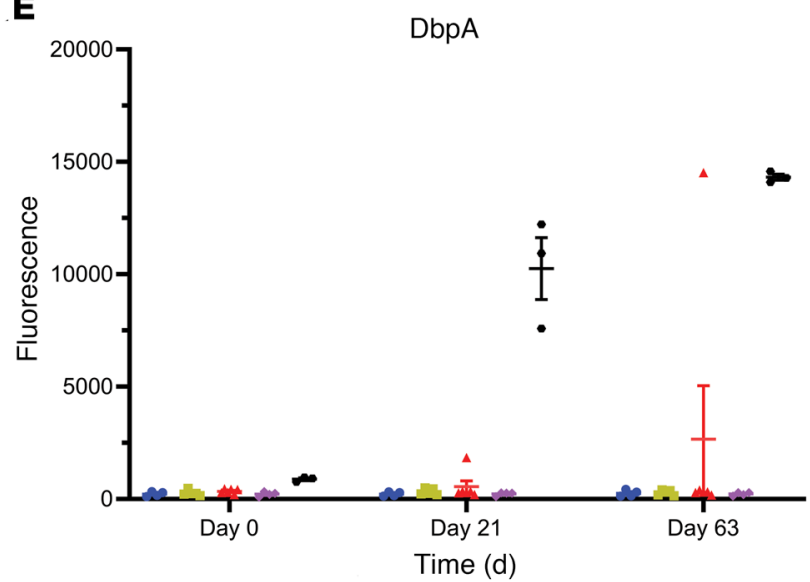

B

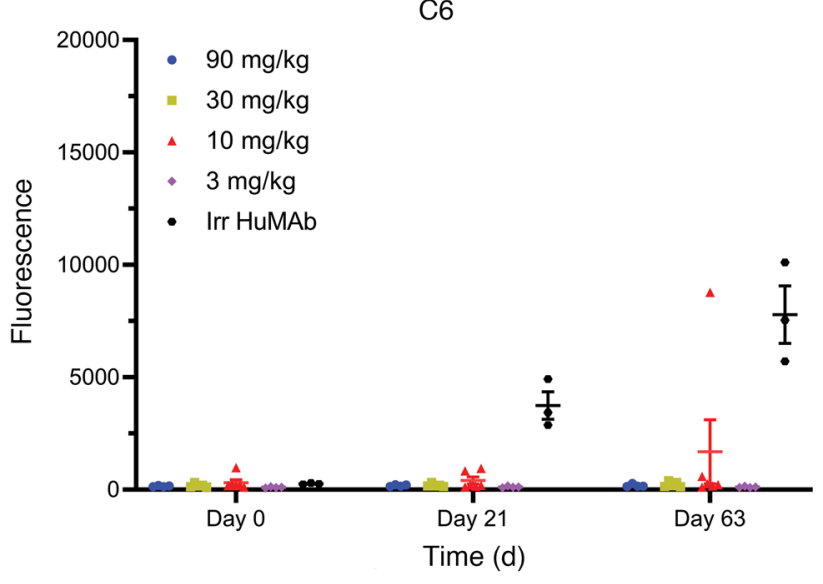

D

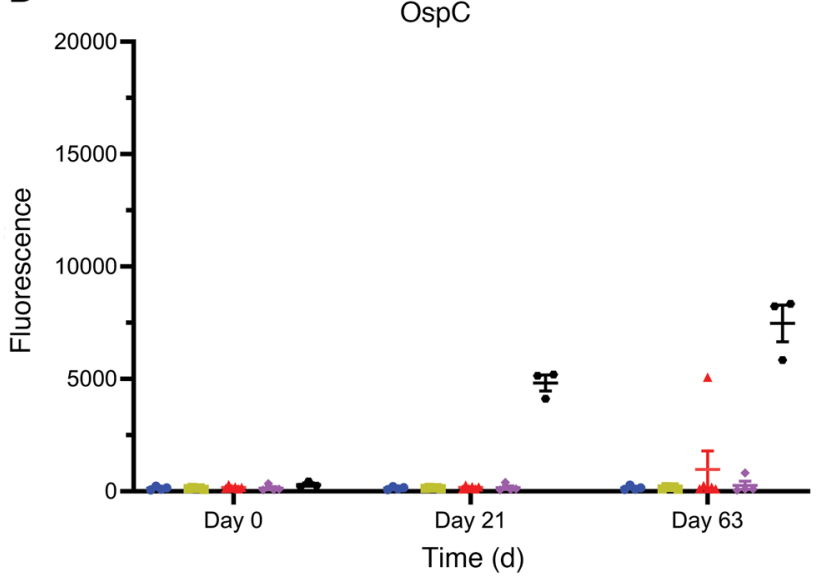

$\mathbf{F}$

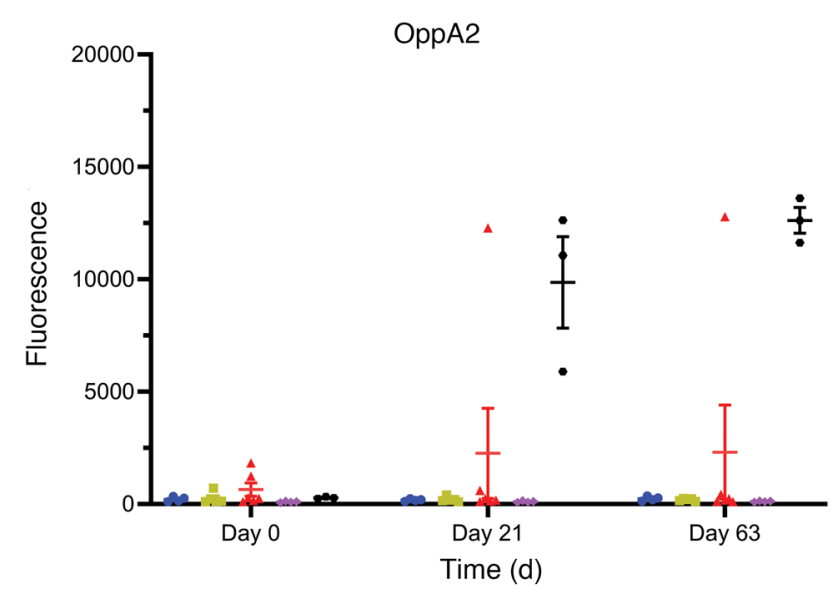

Figure 4. 2217LS blocks transmission of Borrelia burgdorferi in a nonhuman primate model. Seroconversion for lgG antibodies against the $B$. burgdorferi antigen $\mathrm{C} 6$ were measured by ELISA to analyze transmissions as an indicator of efficacy (A). Only 1 animal, in the $2217 \mathrm{LS} 10 \mathrm{mg} / \mathrm{kg}$ group (red), showed evidence of transmission by an increase in anti-C6 IgGs by 21 days after tick exposure, which mirrored the seroconversion of irrelevant lgG-treated animals. All remaining animals in the $3 \mathrm{mg} / \mathrm{kg}$ (purple), $10 \mathrm{mg} / \mathrm{kg}$ (red), $30 \mathrm{mg} / \mathrm{kg}$ (yellow), and $90 \mathrm{mg} / \mathrm{kg}$ (blue) groups were protected, with no detectable $/ g \mathrm{C}$ response against the $\mathrm{C} 6$ peptide, indicating protection levels of $100 \%$ with the exception of $83 \%$ for $10 \mathrm{mg} / \mathrm{kg}$. Symbols represent the mean for each animal tested in $n=3$ independent assays. ELISA results were confirmed by 5 -antigen fluorescent bead-based assay using C6 (B), OspA (C), OspC (D), DbpA (E), and OppA2 (F) antigens. Data are plotted as the mean \pm SD of $n=3$ independent assays. 
A

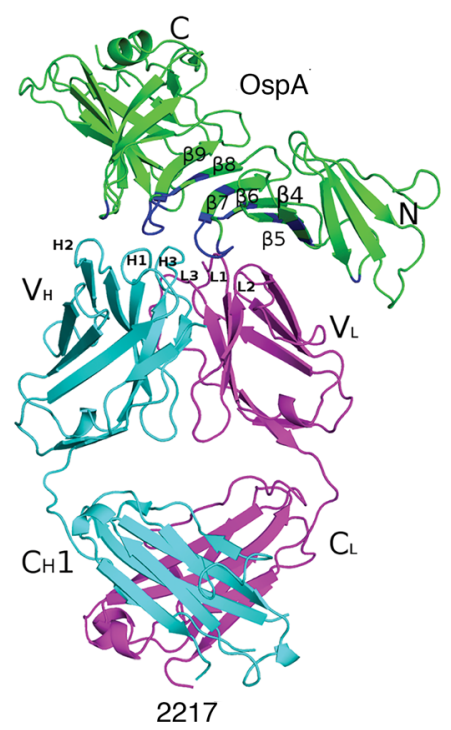

C

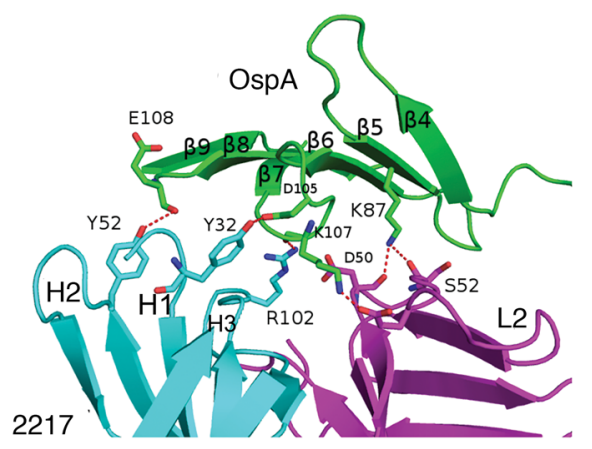

B
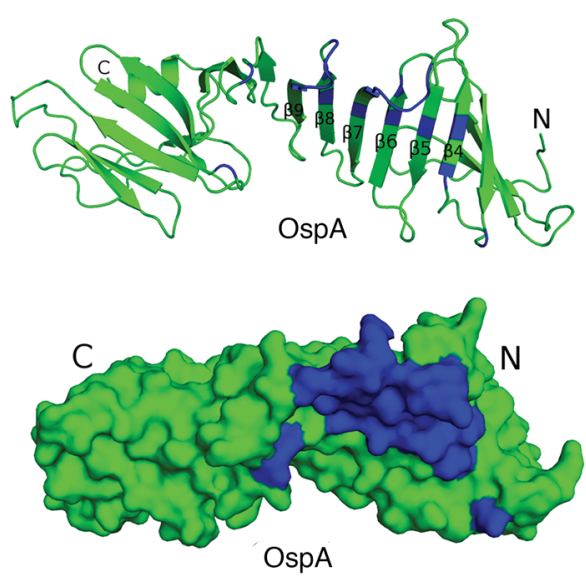

D

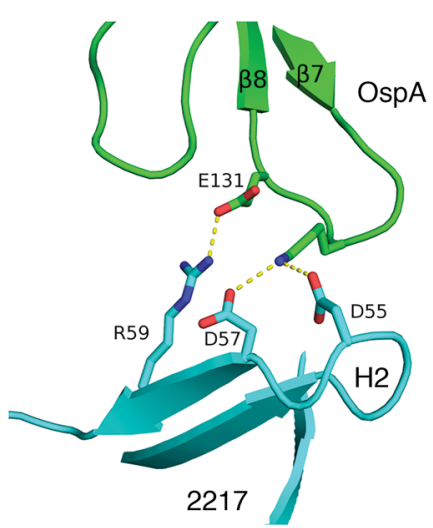

Figure 5. Structure of OspA-2217 complex. (A) The structure of OspA is colored green in complex with Fab 2217; the Fab heavy chain $\left(V_{H}\right.$ and $\left.C_{H} 1\right)$ is colored cyan; the light chain $\left(V_{L}\right.$ and $\left.C_{L}\right)$ is colored magenta; all 3 are depicted as ribbon diagrams. Light and heavy chain CDRs 1, 2, and 3 are labeled L1, L2, and $\mathrm{L} 3$ and $\mathrm{H} 1, \mathrm{H} 2$, and $\mathrm{H} 3$, respectively. OspA residues that bind Fab 2217 are colored blue, with $\beta$-strands 4-9 labeled. (B) OspA-interacting residues with OspA drawn alone (green) displaying the 2217-binding residues colored blue and depicted as a ribbon diagram. Below is OspA in the exact same orientation as the ribbon diagram above drawn as a green surface; the blue surface area highlights the OspA epitope with Fab 2217. Close-up of representative interactions between OspA (green) and Fab 2217 depicting (C) $\mathrm{H}$-bonds and (D) salt bridges. The 2217 Fab heavy chain is colored cyan and the Fab 2217 light chain is colored magenta. All side chains are drawn as sticks and color coordinated to the main chain color with nitrogen atoms shown in blue and oxygen atoms in red. Hydrogen bonds are represented as red dashes in $\mathbf{C}$ and salt bridges are represented as yellow dashes in $\mathbf{D}$. molecule. OspA contained 1 antiparallel $\beta$-sheet composed of 20 antiparallel $\beta$-strands (referred to as $\beta$-strands 1 to 20 ) connecting globular $\mathrm{N}$ - and $\mathrm{C}$-terminal domains followed by a single $\alpha$-helix (referred to as $\alpha$-helix A) (Figure 5B). The OspA structure bound to Fab 2217 was very similar to the OspA-alone structure (Protein Data Bank [PDB] ID 2G8C), with an RMSD of $1.1 \AA$ between 244 residues within each molecule upon superposition, demonstrating no major conformational changes in OspA when bound to Fab 2217 relative to the unbound form of OspA.

Interaction between 2217 and OspA. Altogether, the interaction between Fab 2217 and OspA buried a total surface area of 1440 $\AA^{2}$, generating 7 hydrogen bonds and 5 salt bridges. Five of the 7 hydrogen bonds formed between $2217 \mathrm{H} 1$ residue Tyr-32 and OspA Asp-105, H2 residue Tyr-52 and OspA residue Glu-128, H3 residue Arg-92 and OspA residue Glu-128, H3 residue Arg-102 and OspA residue Lys-107, and $\mathrm{H} 3$ residue Tyr-103 and OspA residue Glu-124. The 2 hydrogen bonds formed between $2217 \mathrm{~V}_{\mathrm{L}}$ domain and OspA involved L2 residues Asp-50 and Ser-52 with Lys-87 from OspA (Figure 5C). The 4 salt bridges formed between 2217 H2 residues Asp-55 and Asp-57 with OspA residue Lys-129, $\beta$-strand residue Arg-59 and OspA's Glu-131, and residue Asp109 with OspA's Lys-107. Fab 2217's $\mathrm{V}_{\mathrm{L}}$ domain participated in the fifth salt bridge between L2 residue Glu-55 and Lys-107 from
OspA (Figure 5D). Overall, the interface between Fab 2217 and OspA was dominated by the $2217 \mathrm{~V}_{\mathrm{H}}$ domain, which contributed $1038 \AA^{2}$ to the total buried surface area while participating in 5 of the 7 hydrogen bonds and 4 of the 5 salt bridges. All 62217 CDRs interacted with the central region of OspA's elongated $\beta$-sheet; $\mathrm{H} 1, \mathrm{H} 2, \mathrm{H} 3$, and $\mathrm{L} 2$ contributed most to the interaction; L1 with L3 made minor contributions to the interface with OspA. More specifically, L1-L3 contacted residues 68 within the loop segment between $\beta$-strands 3-4; residues 75, 77, 87, 92, 100, 102, and 112 in $\beta$-strands 4-7; and 106-107 and 109 within the loop segment between $\beta$-strands 6-7. L1 also associated with Lys-193 from the loop between $\beta$-strands $14-15$ in OspA. H1-H3 made connections with OspA residues 100, 102, 112, 124, 126, and 132 in $\beta$-strands 6-9 along with residues $105,107-109,128-132$, and 152 from the loops between $\beta$-strands 6-7, 8-9, and 9-10, respectively. $\mathrm{H} 3$ also contacted Lys-193 within the loop between $\beta$-strands $14-15$ in OspA (Figure 5, A and B).

\section{Discussion}

We demonstrated that 2217LS, a potent half-life-extended HuM$\mathrm{Ab}$, was capable of protecting nonhuman primates from transmission of spirochetes at a dose as low as $3 \mathrm{mg} / \mathrm{kg}$ when administered prior to challenge with 20 B. burgdorferi-infected ticks. The 
administration of a protective antibody with half-life extension could be an effective strategy for preexposure prophylaxis against Lyme disease.

The feasibility of using human mAbs for preexposure prophylaxis has been shown with palivizumab for preventing respiratory syncytial virus (RSV) infection in premature infants. In this pediatric setting, a passively administered $\mathrm{mAb}$ is both safe and effective and provides immediate immunity of known titer, specificity, and duration. Here, we explored a similar strategy to be employed to prevent Lyme disease infections. Preexposure prophylaxis with 2217LS could be administered to individuals living in or visiting Lyme-endemic areas. One challenge of such a strategy for Lyme disease prevention is to ensure that the antibody offers prolonged protection throughout the tick feeding season. To extend serum half-life of 2217, double aa substitutions (LS: M428L and N434S) were introduced into the $\mathrm{Fc}$ domain of 2217 to increase the binding affinity for the neonatal $\mathrm{Fc}$ receptor. This modification led to a 2-fold increase in half-life in cynomolgus monkeys compared with the non-LS antibody, which is comparable to the pharmacokinetics of VRC01LS, an LS-modified broadly neutralizing anti-HIV antibody. The VRC01 LS mutant had a 3-fold longer serum halflife (11.8 days) than WT VRC01 (4.65 days) in nonhuman primates. The pharmacokinetic profile of VRC01 was determined recently in a phase I human study as a half-life of $71 \pm 18$ days over the 5-40 $\mathrm{mg} / \mathrm{kg}$ i.v. dose range (15). Linear regression analysis of 2217LS pharmacokinetics provided a correlation between the observed half-lives in FcRn-transgenic mice, in nonhuman primates, and the known human half-lives of LS-modified antibodies. The resulting human half-life projections indicated that the LS mutation extended the half-life of 2217LS by 2-fold, which is consistent with other LS studies. Of note, our 2217 WT antibody had a longer half-life of 12 days compared with 4.65 days for VRC01 WT (16). The LS mutant resulted in a 2-fold increase of half-life of 31 days in nonhuman primates. Thus, the half-life of 2217 LS in humans is likely to be similar to or better than VRC01.

Our results established that 2217LS pharmacokinetics in primates were comparable to the pharmacokinetics of other LS HuMAbs in primates that also have human pharmacokinetics data. This similarity allowed us to generally estimate the pharmacokinetics we might observe in the clinic using modeling based on these other LS HuMAbs. Our estimates served the design of the phase I study, but the actual 2217LS human pharmacokinetics and immunogenicity can only be determined from human studies, whether a single dose or multiple doses. Further, we demonstrated that the presence of 2217LS in the blood of the animal prevented transmission of B. burgdorferi from infected ticks to the host. The rhesus macaque model was chosen for the challenge studies because these primates recapitulate the early and disseminated phases of human Lyme disease, including EM and IgG and IgM responses $(18,22)$. The study was designed with an acute challenge because an acute challenge model reduces the potential effects that a nonhuman primate antihuman antibody response could have on antibody efficacy. These responses typically develop 2 weeks after administration, which was the case with 2217LS as well as the irrelevant human IgG antibody. Our results are consistent with other human antibodies tested in nonhuman primate models, and analysis has shown limited correlation between nonhuman primate antidrug responses and human antidrug responses with the same antibodies (23). Additionally, by administering different doses of 2217LS to the animals and measuring the antibody concentration immediately prior to tick challenge, we were able to estimate a range of 2217LS serum concentrations that provide complete protection from the transmission of $B$. burgdorferi by infected ticks to a primate host. The range of protective 2217LS serum concentrations determined by this study needs to be combined with 2217LS human pharmacokinetic data to generate the model necessary for estimating a range of human doses that would result in serum concentrations above the minimum protective level for the 6- to 8-month target window.

The rhesus model has limitations. The infectivity of spirochetes delivered by ticks appears to depend on the blood meal. We do not have infectivity data from capillary-fed nymphs, but one study indicated that the $\mathrm{ID}_{50}$ for mice with spirochetes from fed ticks was approximately 30 organisms (24). Studies also showed that resting nymphs (4 weeks after molting from blood meal) plateau at roughly $1 \times 10^{4}$ spirochetes per tick (25). However, a capillary feeding method, not a blood meal, was used to infect the nymphs for this study. Infection was confirmed after the highdose delivery, but the number of infectious spirochetes at the time of infection was not enumerated. The untreated control animals served to demonstrate the infectivity of spirochetes transmitted from ticks, all of which were derived from the same cohort.

Additionally, compared with mice, the spirochete burden is very low and the size of primates makes direct detection immensely challenging. The ability to culture spirochetes from skin biopsies proved to be highly variable. In the absence of an EM rash, the biopsies were taken near the papules that serve as evidence of the tick bite site. In a human with EM, the biopsy would be taken from the leading edge. Without the EMs, the skin sample collection might not reflect the dispersion of spirochetes, leading to negative culture results. This is in agreement with previous studies showing the ability to assess direct infection in primates using skin biopsies is not overly reliable and needs the support of other methods, including xenodiagnoses, PCR, RT-PCR, and immunofluorescence of necropsy tissues $(26,27)$. Therefore, serology should be weighed more heavily than skin biopsy for diagnosis of breakthrough events.

Analysis of the crystal structure of Fab 2217 in complex with OspA revealed that 2217 recognized a conformational epitope on OspA relative to the 2 previously described structures of Fab 184.1 (PDB ID 1OSP) (28) and Fab LA-2 (PDB ID 1FJ1) (29) in complex with OspA (Supplemental Figure 2, A and B). Fab 184.1 interacted principally with the $\mathrm{N}$-terminal domain of OspA, a nonprotective epitope that is membrane tethered in the spirochete. In contrast, LA-2 interacted with the C-terminal domain of OspA located approximately $81 \AA$ away from the membrane-tethered region, which makes it more accessible for protective antibody interaction. Fab 2217 binds the central $\beta$-sheet region of OspA, which is different from the epitopes of both LA-2 and 184.1. Although both LA-2 and 2217 ostensibly interact with accessible regions, 2217 binds to more conserved areas of OspA. We have previously shown that 2217 is a potent antibody with cross-borreliacidal activity against all 3 main genospecies, including B. burgdorferi, B. afzelii, and B. garinii, for Lyme disease (10). The sequence similarity for OspA 
residues that interact with 2217 is approximately $91 \%$ across the 3 main B. burgdorferi sensu lato complex genospecies (Supplemental Figure 2C). In fact, only one B. burgdorferi OspA residue, Glu-131, which contacts 2217 , is not conserved in B. garinii alone where the equivalent residue to Glu-131 is a positively charged lysine residue. The introduction of a positively charged lysine residue within this region of OspA would seemingly diminish 2217's interaction with B. garinii OspA, ultimately lowering the borreliacidal activity of 2217 toward B. garinii. In the 2217-OspA structure, Glu-131 forms a salt bridge with Arg-59 in 2217's H2 (Figure 5C). The replacement of Glu-131 in B. garinii's OspA with a lysine residue would prevent formation of a salt bridge with Arg-59 in 2217. The lysine residue replacing Glu-131 in B. garinii's OspA would likely form a different salt bridge with the nearby Asp-57 in 2217, compensating for the lost salt bridge between Glu-131 and Arg-59 (Figure 5C). More importantly, our previous paper, in which we discuss the discovery of 2217, has shown that 2217 antibodies do not overlap with the arthritogenic epitope (aa 165-173) on OspA that is potentially cross-reactive with $\mathrm{T}$ cells (10). The crystal structure data in the current work verify the epitope location.

In summary, we have developed a half-life-extended OspA-specific HuMAb that is capable of preventing tick transmission of Borrelia infection in nonhuman primates. Preclinical development has been completed, and an ongoing phase I clinical trial has been initiated to evaluate human pharmacokinetics, safety, and tolerability. Given that a conventional vaccine may require multidose administration during the tick season, if the in vivo activity and extended half-life of 2217LS demonstrated in these animal studies translates well to humans, a single administration of 2217LS may provide immediate protection without age or patient limitations in Lyme-endemic regions for the duration of the tick season. Although the nonhuman primates were administered 2217LS by i.v. infusion, our goal is to formulate 2217LS for delivery by s.c. injection, which requires much simpler infrastructure with less medical personnel time and cost. If successful, 2217LS could be administered in doctors' offices, local pharmacies, etc., as needed for one-time visits to high-risk areas, analogous to standard practice for hepatitis A prevention; or once per season, analogous to influenza vaccination. Currently, s.c. administration of 2217LS is undergoing evaluation in the phase I study. The cost of goods of 2217LS is likely to be less than currently licensed HuMAbs because of the increased scale of manufacturing necessary for a large, healthy population versus manufacturing for much smaller populations with specific diseases. As a class, HuMAbs have an excellent safety record, exemplified by palivizumab, indicated for the prevention of serious lower respiratory tract disease caused by RSV in pediatric patients (30). Palivizumab is seasonally and repeatedly administered. The authors expect that the risk of immunogenicity associated with 2217LS would be low, consistent with currently licensed therapeutic mAbs. With the latter, small percentages of patients develop antidrug responses, despite most being repeatedly administered, many via s.c. injection. If present, ADAs generally have little clinically significant interference with the therapeutic activity of HuMAbs. Because 2217LS is targeted for a healthy population, it is imperative that the risk-benefit profile be appropriate for its intended use. Although the class safety and immunogenicity are good, only human studies can determine the risk-benefit profile of 2217LS. We are planning to address these important questions in our ongoing and planned clinical trials.

\section{Methods}

Antibody cloning, expression, and purification. The heavy and light chain genes of 2217LS were cloned into a mammalian expression vector. CHO-S cells (Invitrogen, R80007) were electroporated with the 2217LS expression vector, and transfectants were selected using $50 \mu \mathrm{M}$ methionine sulfoximine. Transformants were screened for antibody expression and the highest expressing clone (>1 g/L) was selected for antibody production in bioreactors. Culture supernatant from bioreactors was clarified using flocculation and filtration followed by purification using protein A followed by ion exchange chromatography. Antibody was formulated at $100 \mathrm{mg} / \mathrm{mL}$ in 10 $\mathrm{mM}$ histidine, $200 \mathrm{mM}$ trehalose, $10 \mathrm{mM}$ methionine ( $\mathrm{pH}$ 5.5) with $0.05 \%$ Tween 80 and was determined to be more than $98 \%$ antibody monomer-lacking detectable endotoxin.

The heavy and light chain genes of 2217 and an irrelevant IgG isotype control directed against a non- $B$. burgdorferi target were cloned into mammalian expression vectors. ExpiCHO-S cells (Invitrogen, A29127) were transiently transfected with either expression vectors. Antibodies were purified from culture supernatant by fast protein liquid chromatography using a protein A column followed by sizeexclusion chromatography. Purified antibody was dialyzed against PBS and concentrated using an Amicon YM-30 Centriprep concentrator as described by the manufacturer. Purified antibody was filter sterilized and protein concentration determined by spectrophotometry.

Expression and purification of OspA. The nucleic acid sequences of OspA from B. burgdorferi B31 (NP_045688) were cloned into a pET45-His vector in frame with a histidine tag. Primers were designed to remove the native signal sequence (aa 1-18) so that it would be expressed as a cytoplasmic protein with improved solubility. The cloned OspA construct was transformed into BL21-DE3 E. coli bacteria (Invitrogen, C600003), and expression was induced with $1 \mathrm{mM}$ isopropyl- $\beta$-D-1-thiogalactopyranoside (IPTG). Bacteria were lysed, and proteins were purified with Ni-NTA agarose beads (Invitrogen) and eluted with $250 \mathrm{mM}$ imidazole (Sigma-Aldrich).

Borreliacidal assay by Bac-Titer Glo detection. Serial dilutions of antibodies were made in $100 \mu \mathrm{L}$ of BSK-H medium containing $10 \%$ guinea pig complement (Sigma-Aldrich) in a Nunc Edge 96-well plate (Thermo Fisher Scientific). A total of $100 \mu \mathrm{L}$ of B. burgdorferi B31 culture at a concentration of $5 \times 10^{6}$ spirochetes $/ \mathrm{mL}$ was added to each well to mix with antibodies. The 96 -well plate was incubated at $37^{\circ} \mathrm{C}$ for 3 days. The spirochete viability was quantified by luciferase detection with Bac-Titer Glo reagent (Promega) and read in a Victor3 multilabel counter (Applied Biosystems). Assays were performed in triplicate. The resulting fluorescence was plotted, and the $\mathrm{EC}_{50}$ and SD were calculated using GraphPad Prism v8.1.1.

FcRn-transgenic murine model. Pharmacokinetics were assessed using a previously described FcRn-transgenic mouse model (17). Hemizygous B6.Cg-Fcgrttm1Dcr Tg(FCGRT)32Dcr/DcrJ mice obtained from The Jackson Laboratory (stock 014565) lacking the endogenous mouse FcRn $\alpha$ gene and expressing the human FcRn $\alpha$ gene on 1 allele under control of the native FcRn promoter were used for this study. Mice received a single i.p. injection containing a mixture of mAbs administered at $1 \mathrm{mg} / \mathrm{kg}$ per antibody. Each mixture was composed of the 5 mAbs (denosumab, actoxumab, bezlotoxum- 
ab, RAB1, and HCV1) with known human half-life and either 2217 or 2217LS (17). Serial bleeds were collected at 2 hours, 1 day, 7 days, 14 days, 21 days, 29 days, and 41 days after injection. Antibody concentration was determined by antigen-specific binding ELISA. Half-life calculations were determined using Phoenix WinNonlin (Certara). Consistent time points representing the $\beta$-phase half-life (7-41 days after injection) were analyzed by the linear trapezoidal rule using linear interpolation. Linear regression analysis provided a correlation between the observed in vivo FcRn-transgenic mouse half-life and the known human half-lives.

Mouse efficacy model. The mouse challenge model has been previously described (12). Briefly, infected ticks were prepared by placing Ixodes larva on B. burgdorferi strain N40-infected mice with severe combined immunodeficiency for a blood meal. Larvae were harvested and allowed to molt to the nymphal stage before use for challenge. Groups of $5 \mathrm{C} 3 \mathrm{H} / \mathrm{HeJ}$ mice (The Jackson Laboratory, stock 00659) were i.p. injected with a HuMAbs at $10 \mathrm{mg} / \mathrm{kg}$. The following day, mice were challenged by the placement of 6 infected tick nymphs behind the ear of each mouse. Three weeks after the tick placement, mice were euthanized, and tissue samples from an ear, the bladder, the heart, and a joint were harvested for culture. Tissue samples were monitored twice weekly for 4 weeks by dark-field microscopy for evidence of growth of spirochetes. Samples were also analyzed for $B$. burgdorferi DNA using OspA-specific PCR analysis. Serum samples were collected on day 21 to analyze the antibody concentration and for serological investigation by ELISA against lysate of B. burgdorferi. Animals were considered uninfected if results of all 3 tests were negative. Protocols were approved by the IACUC of Tufts University.

Nonhuman primate pharmacokinetic model. To determine whether the Fc mutations extended antibody half-life, 8 male cynomolgus monkeys (Macaca fascicularis) of Chinese origin (Alpha Genesis) were divided into 2 treatment groups (Supplemental Table 3). Animals were i.v. injected with $2217(n=4)$ or 2217LS $(n=4)$ at $10 \mathrm{mg} / \mathrm{kg}$. Serum was then collected at days $0,8,15,22,29,43,58,72$, and 86 . Serum antibody concentration and ADA responses were assessed by ELISA. Serum concentrations were analyzed by NCA with manual selection of all time points (1-86 days after injection) using Phoenix WinNonlin (Certara). Half-lives are reported in days and hours as mean \pm SD. Animals that developed an ADA response as defined as an absorbance value greater than 3 standard deviations from the day -7 mean were excluded. All in vitro and in vivo research experiments were repeated at least 2 times, unless otherwise stated within the figure legends.

Tick challenge experiment in rhesus macaques. Twenty-two rhesus macaques (M. mulatta) of Chinese origin (Alpha Genesis/ENVIGO) with an age range of 3-4 years were used in this study. Fourteen of the animals were females and the remaining 8 were males (Supplemental Table 4). One week prior to antibody injection, serum was collected from all animals and screened for anti-2217/anti-2217LS antibodies and IgG responses to B. burgdorferi. Animals were then divided into 5 treatment groups based on equal sex ratios, weight, and pretreatment ADA response when applicable. All animal studies were blinded such that animal caretakers and investigators were blind to the allocations of treatments. Groups 1-4 received 2217LS administered at $90 \mathrm{mg} / \mathrm{kg}$ $(n=4), 30 \mathrm{mg} / \mathrm{kg}(n=5), 10 \mathrm{mg} / \mathrm{kg}(n=6), 3 \mathrm{mg} / \mathrm{kg}(n=4)$; group 5 received an irrelevant HuMAb IgG at $10 \mathrm{mg} / \mathrm{kg}(n=3)$. Infected ticks were generated by the Vector Borne Diseases Core at the Tulane National Primate Research Center using capillary feeding of Ixodes scapularis nymphs with low-passage B. burgdorferi strain B31.5A19 (19, 31). The density of the culture used to feed the ticks was $6 \times 10^{7}$ to 7 $\times 10^{7}$ spirochetes $/ \mathrm{mL}$. The ticks were fed up to a maximum of $5 \mu \mathrm{L}$, resulting in approximately 325,000 spirochetes per tick.

On day 0 , antibodies were i.v. administered followed by the placement of the tick containment system at the back of the animals. Briefly, the containment feeding system is based on a modified LeFlap maggot cage dressing that has been shown to be feasible and reproducible in nonhuman primate studies $(19,27)$. Briefly, the system consists of a dual-layer containment device with a hydrocolloid layer and adhesive-lined polyester layer that can hold 10-20 unfed nymphs. The device is structurally supported by adhesive foam cushioning. Prior to attaching the system to the animal, an area (approximately $25 \mathrm{~cm}$ $\times 20 \mathrm{~cm}$ ) below the animal scapula was shaved. The device was then attached to the shaved area of the animals using adhesive foam and skin glue. One day later, approximately 20 B. burgdorferi-infected nymphs were added to the device. Waiting a day to add the nymphs allowed for the skin oils to return to the shaved area, which improves the feeding rates of the ticks. The device remained in place for 5 days to allow the nymphs to take a blood meal. After 6 days, the device was removed from the animals and the nymphs were collected.

To determine infectivity, skin biopsies from the infection site were taken once a week for 4 weeks after the nymphs were removed. Each biopsy was placed into BSK-H medium (MilliporeSigma, B8291) and cultured for 6 weeks. The skin biopsy cultures were observed by darkfield microscopy for evidence of spirochetes twice per week. PCR was conducted on the biopsy cultures once per week for 6 weeks using OspA-specific PCR analysis. Cultures and PCR were scored as positive or negative for spirochetes. Serum was collected on days -7, 0, 1, 7, 14, 21, 28,45 , and 63. ELISAs were conducted on the serum samples to determine B. burgdorferi seroconversion, antibody pharmacokinetics, and ADA responses. Animals were considered uninfected if their serum was negative for primate antibodies against B. burgdorferi and if PCR and tissue observation were negative for the presence of B. burgdorferi.

OspA-binding ELISA. Dilutions of purified HuMAbs were tested in ELISA for reactivity against OspA proteins. Briefly, 96-well plates were coated with $100 \mu \mathrm{L}$ of $5 \mu \mathrm{g}$ of WT OspA followed by incubation overnight at $4^{\circ} \mathrm{C}$. The plates were blocked with $1 \%$ BSA with $0.05 \%$ Tween 20 in PBS. Purified antibody diluted in $1 \times$ PBS plus $0.1 \%$ Tween 20 was added to the 96-well plates and incubated for 1 hour at room temperature. Plates were stained with alkaline phosphatase (AP) AffiniPure goat anti-human IgG (Jackson ImmunoResearch, 109055-098, 1:1000 dilution) for 1 hour at room temperature. AP AffiniPure goat anti-mouse IgG (Jackson ImmunoResearch, 115-055-003, 1:1000 dilution) was used to detect the His tag in a separate ELISA to verify protein expression and coating. Plates were developed using p-nitrophenyl phosphate (Thermo Fisher Scientific). Absorbance at an OD of $405 \mathrm{~nm}$ was measured on an Emax precision plate reader (Molecular Devices) using Softmax Pro v4.3.1 LS.

ADA-binding ELISA. ADA ELISAs are designed to detect speciesspecific IgG antibodies produced against the HuMAbs. ADA ELISAs were conducted similar to antigen-binding ELISA with a few modifications. Briefly, 96-well plates were coated with $2 \mu \mathrm{g} / \mathrm{mL}$ of the target HuMAb diluted in PBS followed by incubation overnight at $4^{\circ} \mathrm{C}$. Serum collected from the animals was diluted into blocking buffer and then added to the 96-well plates and titrated with blocking buffer. AP AffiniPure goat anti-mouse IgG (Jackson ImmunoResearch, 115-055-003, 
1:1000 dilution) and anti-rhesus IgG1/3 (Nonhuman Primate Reagent Resource, AB_2819287; $1 \mu \mathrm{g} / \mathrm{mL}$ ) were used for antibody detection. ADA titers were determined by the dilution above mean negative control absorbance value plus 3 SDs.

Seroconversion ELISA. Sera from challenged animals were tested in ELISA for reactivity against whole-cell lysate of B. burgdorferi or recombinant B. burgdorferi C6 peptide (32). For whole-cell lysate, a log-phase culture of $B$. burgdorferi was pelleted and sonicated. A 96-well plate was coated with the lysed B. burgdorferi antigen solution $(1 \mu \mathrm{g} / \mathrm{mL})$ and incubated overnight at $4^{\circ} \mathrm{C}$. For recombinant C6 protein, plates were coated with peptide at $0.5 \mu \mathrm{g} / \mathrm{mL}$. Sera samples from challenged animals were diluted 1:50 in blocking buffer and then titrated 1:2 down a 96-well plate and incubated for 1 hour at room temperature. Mouse antibodies against B. burgdorferi were detected with AP-conjugated anti-mouse IgG secondary antibody; nonhuman primate antibodies were detected with mouse anti-rhesus IgG secondary antibody followed by AP-conjugated anti-mouse IgG secondary antibody. A positive antibody response was defined as an absorbance value greater than the mean absorbance value of the negative control samples plus 3 times the SD.

Multiplex assay for nonhuman primate serology. Sera from challenge animals were tested in a 5-antigen fluorescent bead-based assay for diagnosis of Lyme disease as previously described (21). Briefly, $4 \mathrm{~B}$. burgdorferi antigens, OspA, OspC, DbpA, and OppA2, were expressed as GST fusion proteins, purified, and then coupled with cytometric beads using a Bio-Rad Bio-Plex coupling kit. The C6 peptide was synthesized for amine coupling to beads. Serum samples were diluted 1:200 in sample diluent prior to mixing with the magnetic bead cocktail. Following a standard incubation and washing protocol, specific binding was detected with goat anti-rhesus IgG (H+L) PE 6200-09 (Southern Biotech). Samples were read on a Bio-Plex 200 suspension array system and analyzed using Bio-Plex Manager v6.1 software (Bio-Rad).

Cloning, expression, and purification of OspA and Fab 2217 for structural studies. The PCR amplicon for B. burgdorferi OspA containing residues 18 to 273 was subcloned into the pSUMO expression vector, which contained an N-terminal deca-histidine and SUMO tag. All cloning was performed using a standard ligase-independent cloning protocol. OspA was expressed in E. coli strain BL21 (DE3). The transformed bacteria were grown at $37^{\circ} \mathrm{C}$ in $\mathrm{TB}$ medium and induced at $20^{\circ} \mathrm{C}$ with $0.1 \mathrm{mM}$ IPTG at an $\mathrm{OD}_{600}$ of 0.6 for approximately 16 hours at $20^{\circ} \mathrm{C}$. After induction, cells were harvested and resuspended in $20 \mathrm{mM}$ HEPES pH 7.5 and $150 \mathrm{mM} \mathrm{NaCl}$. The cell suspension was sonicated and centrifuged at 30,000 $\mathrm{g}$ for 30 minutes. After centrifugation, the protein-containing supernatant was purified by nickelaffinity and size-exclusion chromatography on an AKTAxpress system (GE Healthcare), which consisted of a $1 \mathrm{~mL}$ nickel affinity column followed by a Superdex 200 16/60 gel filtration column. The elution buffer consisted of 0.5 M imidazole in binding buffer, and the gel filtration buffer consisted of $20 \mathrm{mM}$ HEPES pH 7.5, $150 \mathrm{mM} \mathrm{NaCl}$, and $20 \mathrm{mM}$ imidazole. Fractions containing OspA (18-273) were pooled and subject to TEV protease cleavage (1:10 weight ratio) for 3 hours at room temperature in order to remove their respective fusion protein tags. The cleaved protein was passed over a $1 \mathrm{~mL} \mathrm{Ni-NTA}$ agarose (QIAGEN) gravity column to remove the added TEV protease, cleaved residues, and uncleaved fusion protein. The 2217 IgG was subjected to papain digestion followed by affinity depletion of the Fc fragment by protein A fast protein liquid chromatography to generate 2217 Fab.
The resulting 2217 Fab was purified to homogeneity by size-exclusion chromatography using a Superdex 200 16/60 gel filtration column. After purification of Fab 2217 and OspA, each protein was mixed in a 1:1 stoichiometry to form a stable complex, and then concentrated to a final concentration of $10 \mathrm{mg} / \mathrm{mL}$ for all crystallization trials.

Crystallization and data collection. Fab-OspA crystals were grown by sitting drop vapor diffusion at $4^{\circ} \mathrm{C}$ using a protein to reservoir volume ratio of 1:1 with total drop volumes of $0.2 \mu \mathrm{L}$. Crystals of the Fab-OspA complex were produced using a crystallization solution containing 100 mM sodium cacodylate pH 6.5, 5\% PEG 8000, and 40\% methylpentanediol. All crystals were flash-frozen in liquid nitrogen after a short soak in the appropriate crystallization buffers supplemented with $25 \%$ ethylene glycol. Data were collected at beamline 8.2.2 at the Advanced Light Source, Lawrence Berkeley National Laboratory. All data were indexed, merged, and scaled using HKL2OOO (33), and then converted to structure factor amplitudes using CCP4 (34).

Structure determination and refinement. The Fab-OspA complex structure was solved by molecular replacement using the program Phaser (35). Molecular replacement calculations were performed using the coordinates of the IL-13 Fab (PDB ID 4HWB) as the search model for Fab 2217 and the OspA coordinates (PDB ID 1FJ1) as the search model for OspA. The resulting phase information from molecular replacement was used for manual model building of the FabOspA model using the graphics program COOT (36) and structural refinement employing the PHENIX package (37). Data collection and refinement statistics are listed in Supplemental Table 2. Molecular graphics were prepared using PyMOL (Schrodinger). The structure generated in this study has been deposited in the PDB (http://www. rcsb.org/pdb/) with ID 7JWG, as described in Supplemental Table 2.

Statistics. All statistical analyses were performed using GraphPad Prism v8.1.1. $\mathrm{EC}_{50}$ values were determined by nonlinear regression using a sigmoidal dose-response fit. Protection comparisons were conducted using a 2-sided Fisher's exact test. Half-life comparisons were conducted by using a 2-sided Student's $t$ test. Significance was determined using a $P$ value of less than 0.05 .

Study approval. These studies were carried out in strict accordance with the recommendations in the Guide for the Care and Use of Laboratory Animals of the NIH. The FcRn-transgenic mouse protocol was approved by the IACUC of the University of Massachusetts Medical School (protocol A-2412). The mouse efficacy protocol was approved by the IACUC of Tufts University Sackler School of Graduate Biomedical Sciences (protocol B2017-36). The primate study protocols were approved by the IACUC of the University of Massachusetts Medical School and Biomere (protocol 18-09) and the US Army Medical Research and Materiel Command Animal Care and Use Review Office.

\section{Author contributions}

ZAS contributed to the investigation, data curation, writing of the original draft, manuscript review and editing, and project administration. MJR contributed to methodology, investigation, formal analysis, data curation, and writing of the original draft. JRT, ME, HSL, AK, and ACT conducted the investigation. AL conducted the investigation and provided resources. SAD performed the investigation, formal analysis, and data curation. CAS contributed to the methodology and investigation. EP, RDC, CG, and FF provided resources. NJM supervised the study and acquired funding. LAC and JSB supervised the study. LTH contributed to the methodology and provided resources. MEE contrib- 
uted to the methodology; wrote, reviewed, and edited the manuscript; and provided resources. MSK wrote, reviewed, and edited the manuscript; supervised the study; and acquired funding. YW contributed to conceptualization, visualization, writing of the original draft, reviewing and editing, and funding acquisition.

\section{Acknowledgments}

The authors would like to thank the entire MassBiologics team for their work, including the departments of product discovery, process development, quality control, and manufacturing for their extensive work to produce 2217LS for these studies in accordance with good manufacturing practices. The authors would also like to thank Mary Jacobs at Tulane University for generat- ing infected ticks and the team at Biomere for their exhaustive effort conducting the nonhuman primate challenge model.

This work was supported by Department of Defense TickBorne Disease Research Program (W81XWH-18-1-0375, PI Wang), NIAID (75N93019C00040, PI/PD Mantis), and Tulane National Primate Research Center base grant (P51OD011104-59, PI Embers).

Address correspondence to: Yang Wang or Mark S. Klempner, 460 Walk Hill Street, Boston, Massachusetts 02126, USA. Phone: 617.474.4091; Email: yang.wang@umassmed.edu. Phone: 617.474.3250; Email: mark.klempner@umassmed.edu.
1. Kugeler KJ, et al. Estimating the frequency of Lyme disease diagnoses, United States, 20102018. Emerg Infect Dis. 2021;27(2):616-619.

2. Schwartz AM, et al. Use of commercial claims data for evaluating trends in Lyme disease diagnoses, United States, 2010-2018. Emerg Infect Dis. 2021;27(2):499-507.

3. Sigal LH, et al. A vaccine consisting of recombinant Borrelia burgdorferi outer-surface protein A to prevent Lyme disease. Recombinant outer-surface protein A Lyme disease vaccine study consortium. N Engl JMed. 1998;339(4):216-222.

4. Golde WT, et al. Reactivity with a specific epitope of outer surface protein A predicts protection from infection with the Lyme disease spirochete, Borrelia burgdorferi. Infect Immun. 1997;65(3):882-889.

5. Johnson BJ, et al. Incomplete protection of hamsters vaccinated with unlipidated OspA from Borrelia burgdorferi infection is associated with low levels of antibody to an epitope defined by $\mathrm{mAb}$ LA-2. Vaccine. 1995;13(12):1086-1094.

6. Schaible UE, et al. Monoclonal antibodies specific for the outer surface protein A (OspA) of Borrelia burgdorferi prevent Lyme borreliosis in severe combined immunodeficiency (scid) mice. Proc Natl Acad Sci U S A. 1990;87(10):3768-3772.

7. Valneva Austria GmbH. Alternative Schedule Study For VLA15, a Vaccine Candidate Against Lyme Borreliosis. https://ClinicalTrials.gov/ show/NCT03970733. Updated: February 16, 2021. Accessed April 23, 2021.

8. Valneva Austria GmbH. Immunogenicity and Safety Study of a Vaccine Against Lyme Borreliosis, in Healthy Adults Aged 18 to 65 Years. Randomized, Controlled, Observer-blind Phase 2 Study. https://ClinicalTrials.gov/show/ NCT03769194. Updated January 27, 2021. Accessed April 23, 2021.

9. Valneva Austria GmbH. Study Assessing the Safety, Immunogenicity and Dose Response of VLA15, A New Vaccine Candidate Against Lyme Borreliosis. https://ClinicalTrials.gov/show/ NCT03010228. Updated October 18, 2019. Accessed April 23, 2021.

10. Wang Y, et al. Pre-exposure prophylaxis with OspA-specific human monoclonal antibodies protects mice against tick transmission of Lyme disease spirochetes. J Infect Dis. 2016;214(2):205-211.
11. Roopenian DC, Akilesh S. FcRn: the neonatal Fc receptor comes of age. Nat Rev Immunol. 2007;7(9):715-725.

12. Zalevsky J, et al. Enhanced antibody halflife improves in vivo activity. Nat Biotechnol. 2010;28(2):157-159.

13. Dall'Acqua WF, et al. Properties of human IgG1s engineered for enhanced binding to the neonatal $\mathrm{Fc}$ receptor ( $\mathrm{FcRn})$. J Biol Chem. 2006;281(33):23514-23524.

14. Robbie GJ, et al. A novel investigational Fc-modified humanized monoclonal antibody, motavizumab-YTE, has an extended half-life in healthy adults. Antimicrob Agents Chemother. 2013;57(12):6147-6153.

15. Gaudinski MR, et al. Safety and pharmacokinetics of the Fc-modified HIV-1 human monoclonal antibody VRC01LS: a Phase 1 open-label clinical trial in healthy adults. PLoS Med. 2018;15(1):e1002493.

16. Ko SY, et al. Enhanced neonatal Fc receptor function improves protection against primate SHIV infection. Nature. 2014;514(7524):642-645.

17. Souders CA, et al. A novel in vitro assay to predict neonatal Fc receptor-mediated human IgG half-life. MAbs. 2015;7(5):912-921.

18. Philipp MT, et al. Early and early disseminated phases of Lyme disease in the rhesus monkey: a model for infection in humans. Infect Immun 1993;61(7):3047-3059.

19. Embers ME, et al. Feeding of ticks on animals for transmission and xenodiagnosis in Lyme disease research. J Vis Exp. 2013;(78):50617.

20. Pachner AR, et al. Detection of active infection in nonhuman primates with Lyme neuroborreliosis: comparison of PCR, culture, and a bioassay. J Clin Microbiol.1998;36(11):3243-3247.

21. Embers ME, et al. Five-antigen fluorescent beadbased assay for diagnosis of Lyme disease. Clin Vaccine Immunol. 2016;23(4):294-303.

22. Crossland NA, et al. Late disseminated Lyme disease: associated pathology and spirochete persistence posttreatment in rhesus macaques. Am J Pathol. 2018;188(3):672-682.

23. van Meer PJ, et al. Immunogenicity of mAbs in non-human primates during nonclinical safety assessment. MAbs. 2013;5(5):810-816

24. Kasumba IN, et al. Virulence of the Lyme disease spirochete before and after the tick bloodmeal: a quantitative assessment. Parasit Vectors.
2016;9:129

25. Pospisilova T, et al. Tracking of Borrelia afzelii transmission from infected Ixodes ricinus nymphs to mice. Infect Immun 2019;87(6):e00896-18.

26. Pachner AR, et al. Inoculation of nonhuman primates with the $\mathrm{N} 40$ strain of Borrelia burgdorferi leads to a model of Lyme neuroborreliosis faithful to the human disease. Neurology. 1995;45(1):165-172.

27. Embers ME, et al. Variable manifestations, diverse seroreactivity and post-treatment persistence in non-human primates exposed to Borrelia burgdorferi by tick feeding. PLoS One. 2017;12(12):e0189071.

28. Li H, et al. Crystal structure of Lyme disease antigen outer surface protein A complexed with an Fab. Proc Natl Acad Sci U S A. 1997;94(8):3584-3589.

29. Ding W, et al. Structural identification of a key protective B-cell epitope in Lyme disease antigen OspA. J Mol Biol. 2000;302(5):1153-1164.

30. Resch B. Product review on the monoclonal antibody palivizumab for prevention of respiratory syncytial virus infection. Hum Vaccin Immunother. 2017;13(9):2138-2149.

31. Purser JE, Norris SJ. Correlation between plasmid content and infectivity in Borrelia burgdorferi. Proc Natl Acad Sci U S A. 2000;97(25):13865-13870.

32. Liang FT, et al. Sensitive and specific serodiagnosis of Lyme disease by enzyme-linked immunosorbent assay with a peptide based on an immunodominant conserved region of Borrelia burgdorferi vlsE. J Clin Microbiol. 1999;37(12):3990-3996.

33. Otwinowski Z, Minor W. Processing of x-ray diffraction data collected in oscillation mode. Methods Enzymol. 1997;276:307-326.

34. Winn MD, et al. Overview of the CCP4 suite and current developments. Acta Crystallogr D Biol Crystallogr. 2011;67(pt 4):235-242.

35. McCoy AJ, et al. Phaser crystallographic software. JAppl Crystallogr. 2007;40(pt 4):658-674.

36. Emsley P, et al. Features and development of Coot. Acta Crystallogr D Biol Crystallogr. 2010;66(pt 4):486-501.

37. Adams PD, et al. PHENIX: a comprehensive Python-based system for macromolecular structure solution. Acta Crystallogr D Biol Crystallogr. 2010;66(pt 2):213-221. 